\title{
Hyperbolicity of the Kidder-Scheel-Teukolsky formulation of Einstein's equations coupled to a modified Bona-Masso slicing condition
}

\author{
Miguel Alcubierre, ${ }^{*}$ Alejandro Corichi, ${ }^{\dagger}$ José A. González, ${ }^{\ddagger}$ Darío Núñez, ${ }^{\S}$ and Marcelo Salgado \\ Instituto de Ciencias Nucleares, Universidad Nacional Autónoma de México, A.P. 70-543, México D.F. 04510, México.
}

(Dated: March, 2003.)

\begin{abstract}
We show that the Kidder-Scheel-Teukolsky family of hyperbolic formulations of the $3+1$ evolution equations of general relativity remains hyperbolic when coupled to a recently proposed modified version of the Bona-Masso slicing condition.

PACS numbers: 04.20.Ex, 04.25.Dm, 95.30.Sf,
\end{abstract}

\section{INTRODUCTION}

The Cauchy problem for general relativity has received renewed interest in the last few years. To a large extent, this interest has been motivated by the realization that the mathematical structure of the evolution equations can have a direct impact on the stability of numerical simulations. Research has concentrated in developing strongly, or even symmetric hyperbolic formulations of the evolution equations of general relativity, as such systems can be shown to be wellposed $[1,2,3,4,5,6,7,8,9,10,11,12,13,14,15]$. This well-posedness implies that one can find numerical discretizations that are stable in the sense that the growth of errors is bounded [16].

A related problem to that of finding well-posed systems of evolution equations is the problem of finding well behaved coordinate systems. In a $3+1$ formulation, this problem reduces to choosing conditions that determine the so-called "gauge" quantities, that is, the lapse function and shift vector. The lapse function determines the slicing of the 4-dimensional spacetime into 3-dimensional spatial hypersurfaces, and the shift vector relates the spatial coordinate systems of nearby hypersurfaces. Our group has recently concentrated in studying slicing conditions that can be written as hyperbolic equations for a time function $T$ whose level surfaces correspond to the members of the foliation [17, 18]. In Ref. [17] we concentrated in the so-called Bona-Masso (BM) family of slicing conditions [3] and studied under which circumstances it avoids different types of pathological behaviors, while in Ref. [18] we proposed a modified version of the BM slicing condition that is well adapted to the evolution of static or stationary spacetimes and to the use of a densitized lapse as the fundamental variable.

Whenever one proposes a new gauge condition, the issue arises of studying if such a condition affects the wellposedness of the system of evolution equations as a whole.

\footnotetext{
*Electronic address: malcubi@nuclecu.unam.mx $\dagger$ Electronic address: corichi@nuclecu.unam.mx

¥Electronic address: cervera@nuclecu.unam.mx

$\S$ Electronic address: nunez@nuclecu.unam.mx

๑Electronic address: marcelo@nuclecu.unam.mx
}

Such an analysis, for example, has been carried out by Sarbach and Tiglio [19] for a generalization of the BM condition and more recently by Lindblom and Scheel [20] for another generalization of the BM condition coupled to a " $\Gamma$-driver" shift condition [21]. In both these cases the analysis was done using multi-parameter first order formulations of the Einstein evolution equations. Here we will consider the Kidder-Scheel-Teukolsky (KST) formulation [15] coupled to the modified BM slicing condition studied in [18].

This paper is organized as follows. In Sec. II we introduce briefly the BM slicing condition and its modified form. Section III describes the KST formulation of the Einstein evolution equations. In Sec. IV we analyze the hyperbolicity of the coupled system of KST evolution equations plus modified BM slicing condition. We conclude in Sec. V.

\section{THE MODIFIED BONA-MASSO SLICING CONDITION}

The BM family of slicing conditions [3] is well known and has been discussed extensively in the literature (see for example [17, 22] and references therein). This slicing condition asks for the lapse function to satisfy the following evolution equation

$$
\frac{d}{d t} \alpha \equiv\left(\partial_{t}-\mathcal{L}_{\beta}\right) \alpha=-\alpha^{2} f(\alpha) K,
$$

with $\mathcal{L}_{\beta}$ the Lie derivative with respect to the shift vector $\beta^{i}, K$ the trace of the extrinsic curvature and $f(\alpha)$ a positive but otherwise arbitrary function of $\alpha$. This condition can be shown to be hyperbolic in the sense that it is equivalent to asking for the time function $T$ to satisfy a generalized wave equation.

In a recent paper [18], we have proposed a modified version of condition (2.1) that keeps many of its important properties but is at the same time well adapted to the evolution of static or stationary spacetimes and also to the use of a densitized lapse as a fundamental variable. We believe that having a slicing condition that is compatible with a static solution is a necessary requirement if one looks for symmetry seeking coordinates of the type discussed by Gundlach and Garfinkle [23] and 
by Brady et.al [24], that will be able to find the Killing fields that static (or stationary) spacetimes have, or the approximate Killing fields that many interesting astrophysical systems will have at late times. This modified BM slicing condition has the form

$$
\partial_{t} \alpha=-\alpha f(\alpha)\left(\alpha K-\nabla_{i} \beta^{i}\right)
$$

with $\nabla_{i}$ is the 3 -covariant derivative associated with $g_{i j}$. One can show that this condition can also be obtained from a generalized wave equation for the time function $T$ and is hence also hyperbolic independently of the Einstein equations.

\section{THE KST FAMILY OF FORMULATIONS OF THE EINSTEIN EVOLUTION EQUATIONS}

The KST family of formulations of the Einstein evolution equations is a multi-parameter, fully first order, system of equations for 30 independent dynamical variables $\left\{g_{i j}, K_{i j}, d_{k i j}\right\}$, where $g_{i j}$ is the spatial metric, $K_{i j}$ the extrinsic curvature, and $d_{k i j}:=\partial_{k} g_{i j}$. Notice that the definition of the $d_{k i j}$ is used only for obtaining initial data, the $d$ 's are then promoted to independent variables and their definition in terms of derivatives of the $g$ 's then becomes a constraint.

If we define $\partial_{0} \equiv\left(\partial_{t}-\mathcal{L}_{\beta}\right) / \alpha$, the system of evolution equations in vacuum can be written as:

$$
\begin{aligned}
\partial_{0} g_{i j} & =-2 K_{i j}, \\
\partial_{0} K_{i j} & =R_{i j}-\left(\nabla_{i} \nabla_{j} \alpha\right) / \alpha-2 K_{i m} K_{j}^{m} \\
& +K K_{i j}+\gamma g_{i j} C+\zeta g^{a b} C_{a(i j) b}, \\
\partial_{0} d_{k i j} & =-2 \partial_{k} K_{i j}-2 K_{i j} \partial_{k} \ln \alpha \\
& +\eta g_{k(i} C_{j)}+\chi g_{i j} C_{k},
\end{aligned}
$$

where $\{\gamma, \zeta, \eta, \chi\}$ are free parameters and

$$
\begin{aligned}
C & :=\left(R-K_{a b} K^{a b}+K^{2}\right) / 2, \\
C_{i} & :=\nabla^{a} K_{a i}-\nabla_{i} K, \\
C_{k i j} & :=d_{k i j}-\partial_{k} g_{i j}, \\
C_{l k i j} & :=\partial_{[l} d_{k] i j},
\end{aligned}
$$

are constraints of the system (the first two are the Hamiltonian and momentum constraints, and the last two are consistency constraints). Notice that since the $d_{k i j}$ are not components of a tensor, their Lie derivative with respect to $\beta^{i}$ should be understood as

$$
\begin{aligned}
\mathcal{L}_{\beta} d_{k i j} & =\beta^{a} \partial_{a} d_{k i j}+d_{a i j} \partial_{k} \beta^{a} \\
& +2 d_{k a(i} \partial_{j)} \beta^{a}+2 g_{a(i} \partial_{j)} \partial_{k} \beta^{a} .
\end{aligned}
$$

The Ricci tensor $R_{i j}$ that appears in the evolution equation for $K_{i j}$ is written in terms of the $d$ 's as

$$
\begin{aligned}
R_{i j} & =\frac{1}{2} g^{a b}\left(-\partial_{a} d_{b i j}+\partial_{a} d_{(i j) b}+\partial_{(i} d_{|a b| j)}\right. \\
& \left.-\partial_{(i} d_{j) a b}\right)+\frac{1}{2}\left[d_{i}^{a b} d_{j a b}+\left(d_{k}-2 b_{k}\right) \Gamma_{i j}^{k}\right] \\
& -\Gamma_{i m}^{k} \Gamma_{j k}^{m}
\end{aligned}
$$

with $d_{k}:=g^{i j} d_{k i j}, b_{k}:=g^{i j} d_{i j k}$ and $\Gamma_{j k}^{i}$ the Christoffel symbols associated with $g_{i j}$. It is important to mention that the system of equations above is not the most general form of the KST system which has 12 free parameters. Here we have considered only the 4 parameters that are related to constraint terms and ignored the 7 parameters that redefine the independent variables and the parameter related to the weight of the prescribed densitized lapse which we will replace with our modified BM slicing condition.

In the original analysis of KST, the system of equations (3.1)-(3.3) was shown to be strongly or even symmetric hyperbolic for certain regions of the parameter space $\{\gamma, \zeta, \eta, \chi\}$, with the lapse replaced by a "densitized lapse" $q$ given by

$$
q:=\ln \left(g^{-\sigma} \alpha\right)
$$

with $g$ the determinant of $g_{i j}$, and $\sigma$ positive (with a preferred value of $1 / 2$ ). The densitized lapse $q$ was assumed to be a prescribed, i.e. a priori known, function of space and time. This condition was later relaxed by Sarbach and Tiglio in [19] where the lapse was instead taken to be an arbitrary function of $g$ such that

$$
\sigma_{\mathrm{eff}}:=\frac{g}{\alpha} \partial_{g} \alpha>0
$$

\section{HYPERBOLICITY OF THE KST FORMULATION COUPLED TO THE MODIFIED BM CONDITION}

We start from the modified BM slicing condition (2.2) which we rewrite as

$$
\partial_{t} \alpha=-\alpha f(\alpha) T
$$

with

$$
T:=\alpha K-\nabla_{m} \beta^{m} .
$$

We now define the first order quantity:

$$
A_{i}:=\frac{\partial_{i} \ln \alpha}{f(\alpha)}
$$

From Eq. (4.1) one can easily show that

$$
\partial_{t} A_{i}=-\partial_{i} T
$$

On the other hand, the derivatives of $\alpha$ that appear in the evolution equation for $K_{i j}$ given in the previous section, Eq. (3.2), can be written in terms of $A_{i}$ as

$$
\frac{\nabla_{i} \nabla_{j} \alpha}{\alpha}=f\left[\partial_{(i} A_{j)}+\left(f+\alpha f^{\prime}\right) A_{i} A_{j}-\Gamma_{i j}^{k} A_{k}\right]
$$

where we have used the fact that $\partial_{i} A_{j}$ is symmetric. Notice now that from the evolution equation for $g_{i j}$, Eq. (3.1), one can also find that

$$
\partial_{t} g=-2 g T
$$


which implies that

$$
\partial_{t} D_{i}=-2 \partial_{i} T
$$

with $D_{i}:=\partial_{i} \ln g$. Comparing equations (4.4) and (4.7) we find

$$
\partial_{t} A_{i}=\frac{1}{2} \partial_{t} D_{i}
$$

Now, from the definition of $d_{k i j}$, we should have $D_{i}=d_{i}$, with $d_{i}$ as defined in the previous section. However, since in the KST formulation the evolution equations for the $d_{k i j}$ are modified by adding multiples of constraints to them, we will generally have $\partial_{t} D_{i} \neq \partial_{t} d_{i}$. Because of this, we propose to modify the evolution equation for $A_{i}$ in the following way

$$
\partial_{t} A_{i}=-\partial_{i} T+F_{i}\left(C, C_{k}, C_{k l m}, C_{k l m n}\right)
$$

From the evolution equation (3.3) for $d_{k i j}$, one can find after some algebra

$$
\begin{aligned}
\partial_{t} d_{i} & =-2 \partial_{i} T+\alpha(\eta+3 \chi) C_{i} \\
& +2 \alpha K^{a b} C_{i a b}+C_{m a}{ }^{a} \partial_{i} \beta^{m} \\
& +\beta^{m} \partial_{m} C_{i a}{ }^{a}
\end{aligned}
$$

which means that if we take

$$
\begin{aligned}
2 F_{i} & =\alpha(\eta+3 \chi) C_{i}+2 \alpha K^{a b} C_{i a b} \\
& +C_{m a}{ }^{a} \partial_{i} \beta^{m}+\beta^{m} \partial_{m} C_{i a}{ }^{a}
\end{aligned}
$$

then we will always have

$$
\partial_{t} A_{i}=\frac{1}{2} \partial_{t} d_{i}
$$

The last equation allows us to define the quantities

$$
Q_{i}:=A_{i}-d_{i} / 2 .
$$

These quantities are then such that $\partial_{t} Q_{i}=0$, that is, they are non-dynamical.

Another way to introduce the $Q_{i}$ is the following: From the modified BM condition and the evolution equation for $g_{i j}$ it is easy to show that

$$
\frac{\partial_{t} \alpha}{\alpha f}=\frac{\partial_{t} g}{2 g}
$$

which one can easily integrate to find

$$
g^{1 / 2}=H\left(x^{i}\right) \exp \int \frac{d \alpha}{\alpha f},
$$

with $H\left(x^{i}\right)$ an arbitrary time-independent function. This shows that if we define

$$
q:=\ln \left(g^{-1 / 2} \exp \int \frac{d \alpha}{\alpha f}\right),
$$

then we will have $\partial_{t} q=0$. Notice that the $q$ defined above is just the generalization of the densitized lapse defined in (3.10) for the case $f \neq 1$. One can now show that the $Q_{i}$ defined through (4.13) are precisely such that $Q_{i}=\partial_{i} q$, and since $q$ is time independent, then so are the $Q_{i}$.

Having introduced the non-dynamical quantities $Q_{i}$, we can rewrite the derivatives of $A_{i}$ appearing in the evolution equation of $K_{i j}$ through the term (4.5) in terms of derivatives of $Q_{i}$ and $d_{i}$. Since the $Q_{i}$ do not evolve, they can be considered as source terms. In this way, the system of evolution equations for $K_{i j}$ and $d_{k i j}$ becomes

$$
\begin{aligned}
\partial_{0} K_{i j} & \sim \frac{1}{2} g^{a b}\left[-\partial_{a} d_{b i j}+(1+\zeta) \partial_{a} d_{(i j) b}\right. \\
& +(1-\zeta) \partial_{(i} d_{|a b| j)}-(1+f) \partial_{(i} d_{j) a b} \\
& \left.+\gamma g_{i j} g^{k l} \partial_{a}\left(d_{k l b}-d_{b k l}\right)\right] \\
\partial_{0} d_{k i j} & \sim-2 \partial_{k} K_{i j}+\eta g_{k(i} g^{a b}\left(\partial_{|a|} K_{j) b}-\partial_{j)} K_{a b}\right) \\
& +\chi g_{i j} g^{a b}\left(\partial_{a} K_{k b}-\partial_{k} K_{a b}\right)
\end{aligned}
$$

where the symbol $\sim$ means equal up to principal part. The system above is exactly the same as the one presented by Sarbach and Tiglio in [19] with the replacement $\sigma_{\text {eff }}=f / 2$. The hyperbolicity analysis of that reference then follows directly. In particular, the non-zero eigenvalues of the system become

$$
\begin{aligned}
& \lambda_{1}=f, \\
& \lambda_{2}=1+\chi-\frac{1}{2}(1+\zeta) \eta+\gamma(2-\eta+2 \chi), \\
& \lambda_{3}=\frac{1}{2} \chi+\frac{3}{8}(1-\zeta) \eta-\frac{1}{4}(1+f)(\eta+3 \chi), \\
& \lambda_{4}=1 .
\end{aligned}
$$

There are 12 eigenvectors associated with these non-zero eigenvalues: two with both $\lambda_{1}$ and $\lambda_{2}$, and four with both $\lambda_{3}$ and $\lambda_{4}$. There are 12 more eigenvectors with eigenvalue zero. The system can be shown to be strongly hyperbolic if

$$
\begin{aligned}
& \lambda_{j}>0, \quad \text { for } j=1,2,3, \\
& \lambda_{3}=\frac{1}{4}\left(3 \lambda_{1}+1\right) \quad \text { if } \lambda_{1}=\lambda_{2} .
\end{aligned}
$$

The associated characteristic speeds are given simply by $v_{i}^{ \pm}= \pm\left(\lambda_{i}\right)^{1 / 2}$. In particular, we obtain $v_{1}^{ \pm}= \pm f^{1 / 2}$, which agrees with the expected result for the BM slicing condition.

\section{DISCUSSION}

We have studied the hyperbolicity of the KST family of formulations of the Einstein evolution equations coupled to a recently proposed modified BM slicing condition. We find that the modified BM condition allows one to construct a non-dynamical function $q$ that generalizes the densitized lapse to the case when the function $f(\alpha)$ defining the slicing is different from 1 . From this nondynamical quantity one can construct three first order 
non-evolving quantities $Q_{i}:=\partial_{i} q$ that can be used to replace the spatial derivatives of the lapse in the evolution equation of the extrinsic curvature $K_{i j}$. By doing this we are able to reduce the system of evolution equations to one previously analyzed by Sarbach and Tiglio, which allows us to show that the coupled KST plus modified BM slicing condition system remains strongly hyperbolic in the same circumstances as before, and also to identify directly the characteristic speeds.

\section{Acknowledgments}

We thank Olivier Sarbach and Manuel Tiglio for many useful comments. This work was supported in part by CONACyT through the repatriation program and grants 149945, 32551-E and J32754-E, by DGAPAUNAM through grants IN112401 and IN122002, and by DGEP-UNAM through a complementary grant.
[1] C. Bona and J. Massó, Phys. Rev. Lett. 68, 1097 (1992).

[2] C. Bona and J. Massó, International Journal of Modern Physics C: Physics and Computers 4, 88 (1993).

[3] C. Bona, J. Massó, E. Seidel, and J. Stela, Phys. Rev. Lett. 75, 600 (1995), gr-qc/9412071.

[4] Y. Choquet-Bruhat and J. York, C. R. Acad. Sc. Paris 321, 1089 (1995).

[5] S. Frittelli and O. Reula, Phys. Rev. Lett. 76, 4667 (1996), gr-qc/9605005.

[6] S. Frittelli and O. Reula, J. Math. Phys. 40, 5143 (1999), gr-qc/9904048.

[7] H. Friedrich, Class. Quantum Grav. 13, 1451 (1996).

[8] M. H. van Putten and D. Eardley, Phys. Rev. D 53, 3056 (1996).

[9] A. Abrahams, A. Anderson, Y. Choquet-Bruhat, and J. York, C.R. Acad. Sci. Paris t. 323, Serie II, 835 (1996).

[10] C. Bona, J. Massó, E. Seidel, and J. Stela, Phys. Rev. D 56, 3405 (1997), gr-qc/9709016.

[11] A. Abrahams, A. Anderson, Y. Choquet-Bruhat, and J. York, Class. Quantum Grav. 14, A9 (1997).

[12] A. Anderson, Y.Choquet-Bruhat, and J. York, Topol. Meth. Nonlinear Anal. 10, 353 (1997).

[13] A. Anderson and J. W. York, Phy. Rev. Lett. 82, 4384
(1999), gr-qc/9901021.

[14] M. Alcubierre, B. Brügmann, M. Miller, and W.-M. Suen, Phys. Rev. D 60, 064017 (1999), gr-qc/9903030.

[15] L. E. Kidder, M. A. Scheel, and S. A. Teukolsky, Phys. Rev. D 64, 064017 (2001), gr-qc/0105031.

[16] G. Calabrese, J. Pullin, O. Sarbach, and M. Tiglio, Phys. Rev. D 66, 041501 (2002), gr-qc/0207018.

[17] M. Alcubierre, Class. Quantum Grav. 20, 607 (2002), gr-qc/0210050.

[18] M. Alcubierre et al., (2003), gr-qc/0303069.

[19] O. Sarbach and M. Tiglio, Phys. Rev. D 66, 064023 (2002), gr-qc/0205086.

[20] L. Lindblom and M. A. Scheel, (2003), gr-qc/0301120.

[21] M. Alcubierre et al., (2002), to be published in Phys. Rev. D, gr-qc/0206072.

[22] M. Alcubierre et al., Phys. Rev. Lett. 87, 271103 (2001), gr-qc/0012079.

[23] D. Garfinkle and C. Gundlach, Class. Quantum Grav. 16, 4111 (1999), gr-qc/9908016.

[24] P. R. Brady, J. D. E. Creighton, and K. S. Thorne, Phys. Rev. D 58, 061501 (1998), gr-qc/9804057. 